\title{
Performance-Based Adaptive Gradient Descent Optimal Coefficient Fuzzy Sliding Mode Methodology
}

\author{
Farzin Piltan \\ Industrial Electrical and Electronic Engineering SanatkadeheSabze Pasargad. CO (S.S.P. Co), NO:16, PO.Code 71347- \\ 66773, Fourth floor, Dena Apr, Seven Tir Ave, Shiraz, Iran \\ Email: SSP.ROBOTIC@gmail.com
}

Bamdad Boroomand

Industrial Electrical and Electronic Engineering SanatkadeheSabze Pasargad. CO (S.S.P. Co), NO:16, PO.Code 7134766773, Fourth floor, Dena Apr, Seven Tir Ave, Shiraz, Iran

Email: SSP.ROBOTIC@yahoo.com

\author{
Arman Jahed \\ Industrial Electrical and Electronic Engineering SanatkadeheSabze Pasargad. CO (S.S.P. Co), NO:16, PO.Code 71347- \\ 66773, Fourth floor, Dena Apr , Seven Tir Ave, Shiraz , Iran \\ Email: SSP.ROBOTIC@yahoo.com
}

\author{
Hossein Rezaie \\ Industrial Electrical and Electronic Engineering SanatkadeheSabze Pasargad. CO (S.S.P. Co), NO:16, PO.Code 71347- \\ 66773, Fourth floor, Dena Apr, Seven Tir Ave, Shiraz, Iran \\ Email: SSP.ROBOTIC@yahoo.com
}

\begin{abstract}
Design a nonlinear controller for second order nonlinear uncertain dynamical systems is the main challenge in this paper. This paper focuses on the design and analysis of a chattering free Mamdani's fuzzy-based tuning gradient descent optimal error-based fuzzy sliding mode controller for highly nonlinear dynamic six degrees of freedom robot manipulator, in presence of uncertainties. Conversely, pure sliding mode controller is used in many applications; it has two important drawbacks namely; chattering phenomenon and nonlinear equivalent dynamic formulation in uncertain dynamic parameter. In order to solve the uncertain nonlinear dynamic parameters, implement easily and avoid mathematical model base controller, Mamdani's performance/error-based fuzzy logic methodology with two inputs and one output and 49 rules is applied to pure sliding mode controller. Pure sliding mode controller and error-based fuzzy sliding mode controller have difficulty in handling unstructured model uncertainties. To solve this problem applied fuzzy-based tuning method to error-based fuzzy sliding mode controller for adjusting the sliding surface gain. Since the sliding surface gain is adjusted by gradient descent optimization method. Fuzzy-based tuning gradient descent optimal error-based fuzzy sliding mode controller is stable model-free controller which eliminates the chattering phenomenon without to use the boundary layer saturation function. Lyapunov
\end{abstract}

stability is proved in fuzzy-based tuning gradient descent optimal fuzzy sliding mode controller based on switching (sign) function. This controller has acceptable performance in presence of uncertainty (e.g., overshoot $=0 \%$, rise time $=0.8$ second, steady state error $=1 \mathrm{e}-9$ and RMS error $=1.8 \mathrm{e}-12$ ).

Index Terms - Gradient Descent Optimal Algorithm, Sliding Mode Controller, Gradient Descent Optimal Fuzzy Sliding Mode Controller, Fuzzy Based Tuning Methodology, Lyapunov Based Stability

\section{Introduction}

Robot manipulator is a collection of links that connect to each other by joints, these joints can be revolute and prismatic that revolute joint has rotary motion around an axis and prismatic joint has linear motion around an axis. Each joint provides one or more degrees of freedom (DOF) [1-15].

Controller is a device which can sense information from linear or nonlinear system (e.g., robot manipulator) to improve the systems performance [3-20]. The main targets in designing control systems are stability, good disturbance rejection, and small tracking error[5, 21-30]. Several industrial robot manipulators are controlled by 
linear methodologies (e.g., Proportional-Derivative (PD) controller, Proportional- Integral (PI) controller or Proportional- Integral-Derivative (PID) controller), but when robot manipulator works with various payloads and have uncertainty in dynamic models this technique has limitations. In some applications robot manipulators are used in an unknown and unstructured environment, therefore strong mathematical tools used in new control methodologies to design nonlinear robust controller with an acceptable performance (e.g., minimum error, good trajectory, disturbance rejection) [31-45].

Sliding mode controller is an influential nonlinear controller to certain and uncertain systems which it is based on system's dynamic model. Sliding mode controller is a powerful nonlinear robust controller under condition of partly uncertain dynamic parameters of system [7, 46-61]. This controller is used to control of highly nonlinear systems especially for robot manipulators. Chattering phenomenon and nonlinear equivalent dynamic formulation in uncertain dynamic parameter are two main drawbacks in pure sliding mode controller [20, 46-61]. The chattering phenomenon problem in pure sliding mode controller and fuzzy sliding mode controller is reduced by using linear saturation boundary layer function but prove the stability is very difficult. The nonlinear equivalent dynamic formulation problem in uncertain system is solved by using fuzzy logic theorem but calculate the optimal sliding surface slope coefficient by trial and error is the main challenge [8]. Gradient descent is a first-order optimization algorithm. Gradient descent works in spaces of any number of dimensions, even in infinite-dimensional ones. In the latter case the search space is typically a function space, and one calculates the Gâteaux derivative of the functional to be minimized to determine the descent direction. The gradient descent can take much iteration to compute a local minimum with a required accuracy, if the curvature in different directions is very different for the given functio. This method is based on resolve the sliding surface slope as well as improve the output performance by Gradient Descent Optimal Algorithm (GDOA) tuning the sliding surface slope coefficient. The sliding surface gain $(\boldsymbol{\lambda})$ of this controller is adjusted off line depending on the iterations. Fuzzy logic theory is used to remove the system's dynamics. To estimate the system dynamics, error-based GDOA fuzzy sliding mode controller is designed. Proposed MIMO errorbased GDOA fuzzy sliding mode controller with saturation function is a chattering free dynamic modelfree controller. This methodology is based on applied fuzzy logic in equivalent nonlinear dynamic part to estimate unknown parameters.

This paper is organized as follows; section 2, is served as an introduction to the sliding mode controller formulation algorithm and its application to control of robot manipulator, dynamic of robot manipulator, proof of stability and fuzzy logic method. Part 3, introduces and describes the methodology (design fuzzy-based tuning gradient descent optimal error-based fuzzy sliding mode controller) algorithms and proves Lyapunov stability. Section 4 presents the simulation results and discussion of this algorithm applied to a robot arm and the final section is describing the conclusion.

\section{Theory}

\subsection{Dynamic Formulation of Robot Manipulator}

Dynamic modeling of robot manipulators is used to describe the behavior of robot manipulator such as linear or nonlinear dynamic behavior, design of model based controller such as pure sliding mode controller which design this controller is based on nonlinear dynamic equations, and for simulation. The dynamic modeling describes the relationship between joint motion, velocity, and accelerations to force/torque or current/voltage and also it can be used to describe the particular dynamic effects (e.g., inertia, coriolios, centrifugal, and the other parameters) to behavior of system[1]. The Unimation PUMA 560 serially links robot manipulator was used as a basis, because this robot manipulator is widely used in industry and academic. It has a nonlinear and uncertain dynamic parameters serial link 6 degrees of freedom (DOF) robot manipulator.

The equation of an $n-D O F$ robot manipulator governed by the following equation $[1,4,15-29,50-61]$ :

$$
M(q) \ddot{q}+N(q, \dot{q})=\tau
$$

Where $\tau$ is actuation torque, $\mathrm{M}(\mathrm{q})$ is a symmetric and positive define inertia matrix, $N(q, \dot{q})$ is the vector of nonlinearity term. This robot manipulator dynamic equation can also be written in a following form [1-3]:

$$
\tau=M(q) \ddot{q}+B(q)[\dot{q} \dot{q}]+C(q)[\dot{q}]^{2}+G(q)
$$

Where $\mathrm{B}(\mathrm{q})$ is the matrix of coriolios torques, $\mathrm{C}(\mathrm{q})$ is the matrix of centrifugal torques, and $\mathrm{G}(\mathrm{q})$ is the vector of gravity force. The dynamic terms in equation (2) are only manipulator position. This is a decoupled system with simple second order linear differential dynamics. In other words, the component $\ddot{q}$ influences, with a double integrator relationship, only the joint variable $q_{i}$, independently of the motion of the other joints. Therefore, the angular acceleration is found as to be [3, 41-61]:

$$
\ddot{q}=M^{-1}(q) .\{\tau-N(q, \dot{q})\}
$$

This technique is very attractive from a control point of view. 


\subsection{Sliding Mode Controller}

Consider a nonlinear single input dynamic system is defined by [6]:

$$
x^{(n)}=f(\vec{x})+b(\vec{x}) u
$$

Where $\mathrm{u}$ is the vector of control input, $\boldsymbol{x}^{(\boldsymbol{n})}$ is the $\boldsymbol{n}^{\text {th }}$ derivation of $x, x=\left[x, \dot{x}, \ddot{x}, \ldots, x^{(n-1)}\right]^{T}$ is the state vector, $\boldsymbol{f}(\boldsymbol{x})$ is unknown or uncertainty, and $\boldsymbol{b}(\boldsymbol{x})$ is of known sign function. The main goal to design this controller is train to the desired state; $\boldsymbol{x}_{\boldsymbol{d}}=$ $\left[x_{d}, \dot{x}_{d}, \ddot{x}_{d}, \ldots, x_{d}^{(n-1)}\right]^{T}$, and trucking error vector is defined by [6]:

$$
\widetilde{x}=x-x_{d}=\left[\widetilde{x}, \ldots, \widetilde{x}^{(n-1)}\right]^{T}
$$

A time-varying sliding surface $\boldsymbol{s}(\boldsymbol{x}, \boldsymbol{t})$ in the state space $\boldsymbol{R}^{\boldsymbol{n}}$ is given by [6]:

$$
s(x, t)=\left(\frac{d}{d t}+\lambda\right)^{n-1} \tilde{x}=0
$$

where $\lambda$ is the positive constant. To further penalize tracking error, integral part can be used in sliding surface part as follows [6]:

$$
s(x, t)=\left(\frac{d}{d t}+\lambda\right)^{n-1}\left(\int_{0}^{t} \widetilde{x} d t\right)=0
$$

The main target in this methodology is kept the sliding surface slope $\boldsymbol{s}(\boldsymbol{x}, \boldsymbol{t})$ near to the zero. Therefore, one of the common strategies is to find input $\boldsymbol{U}$ outside of $\boldsymbol{s}(\boldsymbol{x}, \boldsymbol{t})$ [6].

$$
\frac{1}{2} \frac{d}{d t} s^{2}(x, t) \leq-\zeta|s(x, t)|
$$

where $\zeta$ is positive constant.

$$
\text { If } \mathbf{S}(\mathbf{0})>\mathbf{0} \rightarrow \frac{\mathrm{d}}{\mathrm{dt}} \mathbf{S}(\mathbf{t}) \leq-\zeta
$$

To eliminate the derivative term, it is used an integral term from $\mathrm{t}=0$ to $\mathrm{t}=\boldsymbol{t}_{\text {reach }}$

$$
\begin{array}{r}
\int_{t=0}^{t=t_{\text {reach }}} \frac{d}{d t} S(t) \leq-\int_{t=0}^{t=t_{\text {reach }}} \eta \rightarrow S\left(t_{\text {reach }}\right) \\
-S(0) \leq-\zeta\left(t_{\text {reach }}-0\right)
\end{array}
$$

Where $t_{\text {reach }}$ is the time that trajectories reach to the sliding surface so, suppose $\mathrm{S}\left(t_{\text {reach }}=0\right)$ defined as;

$$
0-S(0) \leq-\eta\left(t_{\text {reach }}\right) \rightarrow t_{\text {reach }} \leq \frac{S(0)}{\zeta}
$$

$$
\text { if } \begin{aligned}
\boldsymbol{S}(\mathbf{0})<0 \rightarrow 0- & S(\mathbf{0}) \leq-\boldsymbol{\eta}\left(\boldsymbol{t}_{\text {reach }}\right) \\
& \rightarrow \boldsymbol{S}(\mathbf{0}) \leq-\zeta\left(\boldsymbol{t}_{\text {reach }}\right) \\
& \rightarrow \boldsymbol{t}_{\text {reach }} \leq \frac{|\boldsymbol{S}(\mathbf{0})|}{\boldsymbol{\eta}}
\end{aligned}
$$

Equation (12) guarantees time to reach the sliding surface is smaller than $\frac{|\boldsymbol{S}(\mathbf{0})|}{\zeta}$ since the trajectories are outside of $S(t)$.

$$
\text { if } S_{t_{\text {reach }}}=S(0) \rightarrow \operatorname{error}\left(x-x_{d}\right)=0
$$

Suppose $\mathrm{S}$ is defined as

$$
\begin{aligned}
s(x, t)=\left(\frac{d}{d t}+\lambda\right) & \tilde{x} \\
& =\left(\dot{\mathrm{x}}-\dot{\mathbf{x}}_{\mathrm{d}}\right)+\lambda\left(\mathbf{x}-\mathbf{x}_{\mathrm{d}}\right)
\end{aligned}
$$

The derivation of $\mathrm{S}$, namely, $\dot{S}$ can be calculated as the following;

$$
\dot{S}=\left(\ddot{\mathbf{x}}-\ddot{\mathbf{x}}_{\mathbf{d}}\right)+\lambda\left(\dot{\mathbf{x}}-\dot{\mathbf{x}}_{\mathbf{d}}\right)
$$

Suppose the second order system is defined as;

$$
\ddot{x}=f+u \rightarrow \dot{S}=f+U-\ddot{x}_{d}+\lambda\left(\dot{\mathbf{x}}-\dot{\mathbf{x}}_{\mathrm{d}}\right)
$$

Where $\boldsymbol{f}$ is the dynamic uncertain, and also since $S=0$ and $\dot{S}=0$, to have the best approximation , $\widehat{\boldsymbol{U}}$ is defined as

$$
\widehat{U}=-\widehat{f}+\ddot{x}_{d}-\lambda\left(\dot{\mathbf{x}}-\dot{\mathbf{x}}_{\mathbf{d}}\right)
$$

A simple solution to get the sliding condition when the dynamic parameters have uncertainty is the switching control law [58-61]:

$$
U_{d i s}=\widehat{U}-K(\vec{x}, t) \cdot \operatorname{sgn}(s)
$$
6]

Where the switching function $\mathbf{s g n}(\mathbf{S})$ is defined as $[1$,

$$
\operatorname{sgn}(s)= \begin{cases}1 & s>0 \\ -1 & s<0 \\ 0 & s=0\end{cases}
$$

And the $\boldsymbol{K}(\overrightarrow{\boldsymbol{x}}, \boldsymbol{t})$ is the positive constant. Suppose by (8) the following equation can be written as,

$$
\begin{aligned}
\frac{1}{2} \frac{d}{d t} s^{2}(x, t)=\dot{S} \cdot S & =[f-\widehat{f}-K \operatorname{sgn}(s)] \cdot S \\
= & (f-\widehat{f}) \cdot S-K|S|
\end{aligned}
$$

And if the equation (12) instead of (11) the sliding surface can be calculated as

and 


$$
\begin{aligned}
s(x, t)=\left(\frac{d}{d t}+\lambda\right)^{2} & \left(\int_{0}^{t} \tilde{x} d t\right) \\
& =\left(\dot{\mathbf{x}}-\dot{\mathbf{x}}_{\mathrm{d}}\right)+2 \lambda\left(\dot{\mathbf{x}}-\dot{\mathbf{x}}_{\mathrm{d}}\right) \\
& -\lambda^{2}\left(\mathbf{x}-\mathbf{x}_{\mathrm{d}}\right)
\end{aligned}
$$

in this method the approximation of $\boldsymbol{U}$ is computed as [6]

$$
\widehat{U}=-\widehat{f}+\ddot{x}_{d}-2 \lambda\left(\dot{\mathbf{x}}-\dot{\mathbf{x}}_{\mathbf{d}}\right)+\lambda^{2}\left(\mathbf{x}-\mathbf{x}_{\mathbf{d}}\right)
$$

Based on above discussion, the sliding mode control law for a multi degrees of freedom robot manipulator is written as $[1,6]$ :

$$
\tau=\tau_{e q}+\tau_{d i s}
$$

Where, the model-based component $\boldsymbol{\tau}_{\boldsymbol{e q}}$ is the nominal dynamics of systems calculated as follows [1]:

$$
\tau_{e q}=\left[M^{-1}(B+C+G)+\dot{S}\right] M
$$

and $\boldsymbol{\tau}_{\boldsymbol{d i s}}$ is computed as [1];

$$
\tau_{d i s}=K \cdot \operatorname{sgn}(S)
$$

By (25) and (24) the sliding mode control of robot manipulator is calculated as;

$$
\tau=\left[M^{-1}(B+C+G)+\dot{S}\right] M+K \cdot \operatorname{sgn}(S)
$$

where $S=\lambda e+\dot{e}$ in $\mathrm{PD}-\mathrm{SMC}$ and $S=\lambda e+\dot{e}+$ $\left(\frac{\lambda}{2}\right)^{2} \sum e$ in PID-SMC.

\subsection{Proof of Stability}

The lyapunov formulation can be written as follows,

$$
V=\frac{1}{2} S^{T} \cdot M \cdot S
$$

The derivation of $V$ can be determined as,

$$
\dot{V}=\frac{1}{2} S^{T} \cdot \dot{M} \cdot S+S^{T} M \dot{S}
$$

The dynamic equation of robot manipulator can be written based on the sliding surface as

$$
\boldsymbol{M} \dot{\boldsymbol{S}}=-\boldsymbol{V} \boldsymbol{S}+\boldsymbol{M} \dot{\boldsymbol{S}}+\boldsymbol{B}+\boldsymbol{C}+\boldsymbol{G}
$$

It is assumed that

$$
S^{T}(\dot{M}-2 B+C+G) S=0
$$

by substituting (29) in (28)

$$
\begin{array}{r}
\dot{V}=\frac{1}{2} S^{T} \dot{M} S-S^{T} B+C S+S^{T}(M \dot{S}+B+C S+G) \\
=S^{T}(M \dot{S}+B+C S+G)
\end{array}
$$

Suppose the control input is written as follows

$$
\begin{aligned}
\widehat{U}=U_{\text {Nonlenear }}+\widehat{U_{d l s}} & \\
& =\left[\widehat{M^{-1}}(B+C+G)+\dot{S}\right] \widehat{M} \\
& +K \cdot \operatorname{sgn}(S)+B+C S+G
\end{aligned}
$$

By replacing the equation (32) in (31)

$$
\begin{aligned}
\dot{V}=S^{T}(M \dot{S}+B+C & +G-\widehat{M} \dot{S}-\widehat{B+C S}+G \\
& -K \operatorname{sgn}(S) \\
& =S^{T}(\widetilde{M} \dot{S}+\widehat{B+C S}+G \\
& -K \operatorname{sgn}(S))
\end{aligned}
$$

And

$$
|\widetilde{M} \dot{S}+\widetilde{B+C}+G| \leq|\widetilde{M} \dot{S}|+|\widetilde{B+C} S+G|
$$

The Lemma equation in robot arm system can be written as follows

$$
K_{u}=[|\widetilde{M} \dot{S}|+|B+C S+G|+\eta]_{i}, i=1,2,3,4, \ldots
$$

And finally;

$$
\dot{V} \leq-\sum_{i=1}^{n} \eta_{i}\left|S_{i}\right|
$$

Figure 1 is shown pure sliding mode controller applied to robot manipulator.

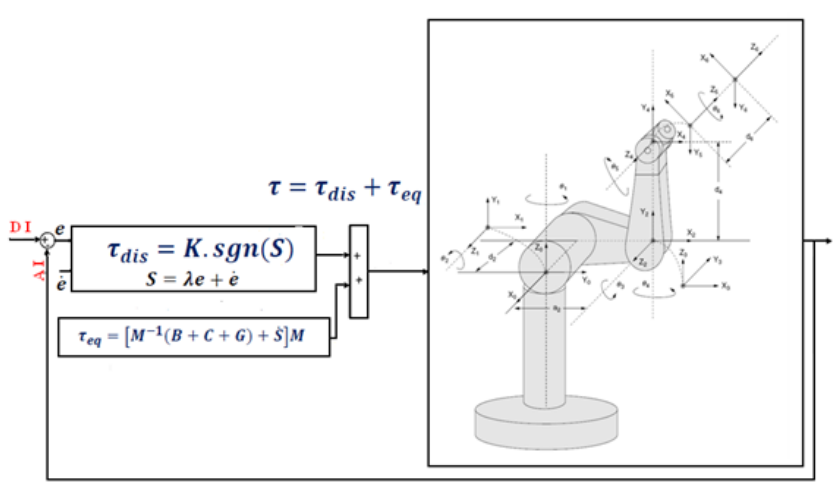

Fig. 1: Sliding Mode Controller

\subsection{Fuzzy Logic Methodology}

Based on foundation of fuzzy logic methodology; fuzzy logic controller has played important rule to design nonlinear controller for nonlinear and uncertain systems [53]. However the application area for fuzzy control is really wide, the basic form for all command types of controllers consists of;

Input fuzzification (binary-to-fuzzy $[\mathrm{B} / \mathrm{F}]$ conversion) 
Fuzzy rule base (knowledge base), Inference engine and Output defuzzification (fuzzy-to-binary [F/B] conversion). Figure 2 is shown a fuzzy controller part.

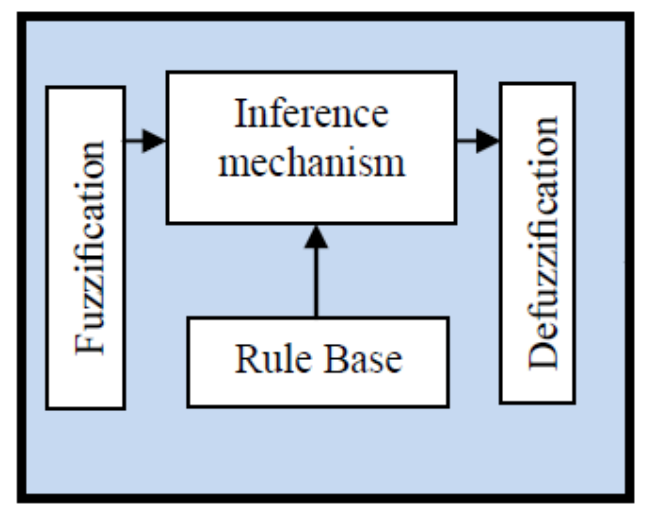

Fig. 2: Fuzzy Controller Part

The fuzzy inference engine offers a mechanism for transferring the rule base in fuzzy set which it is divided into two most important methods, namely, Mamdani method and Sugeno method. Mamdani method is one of the common fuzzy inference systems and he designed one of the first fuzzy controllers to control of system engine. Mamdani's fuzzy inference system is divided into four major steps: fuzzification, rule evaluation, aggregation of the rule outputs and defuzzification. Michio Sugeno use a singleton as a membership function of the rule consequent part. The following definition shows the Mamdani and Sugeno fuzzy rule base

\section{if $x$ is $A$ and $y$ is $B$ then $z$ is C'mamdani' \\ if $x$ is $A$ and $y$ is $B$ then $z$ is $f(x, y)$ 'sugeno'}

When $x$ and $y$ have crisp values fuzzification calculates the membership degrees for antecedent part. Rule evaluation focuses on fuzzy operation $(A N D / O R)$ in the antecedent of the fuzzy rules. The aggregation is used to calculate the output fuzzy set and several methodologies can be used in fuzzy logic controller aggregation, namely, Max-Min aggregation, Sum-Min aggregation, Max-bounded product, Max-drastic product, Max-bounded sum, Max-algebraic sum and Min-max. Defuzzification is the last step in the fuzzy inference system which it is used to transform fuzzy set to crisp set. Consequently defuzzification's input is the aggregate output and the defuzzification's output is a crisp number. Centre of gravity method (COG) and Centre of area method $(C O A)$ are two most common defuzzification methods.

\section{Methodology}

\subsection{Design Fuzzy Sliding Mode Controller}

As shown in Figure 1, sliding mode controller is divided into two main parts: discontinuous part and equivalent part. Discontinuous part is based on switching function which this method is used to have stability but it can caused chattering. Equivalent part is based on robot manipulator's dynamic formulation which these formulations are nonlinear; MIMO and some of them are unknown. Equivalent part of sliding mode controller is based on nonlinear dynamic formulations of robot manipulator. Robot manipulator's dynamic formulations are highly nonlinear and some of parameters are unknown therefore design a controller based on dynamic formulation is complicated. To solve this challenge fuzzy logic methodology is applied to sliding mode controller. Based on literature [43-44, 5861], most of researchers are designed fuzzy modelbased sliding mode controller and model-based sliding mode fuzzy controller. In this method fuzzy logic method is used to estimate some dynamic formulation that they are used in equivalent part. To solve the challenge of sliding mode controller based on nonlinear dynamic formulation this research is focused on eliminate the nonlinear equivalent formulation. In this method; dynamic nonlinear equivalent part is replaced by performance/error-based fuzzy logic controller. In fuzzy error-based sliding mode controller; error based Mamdani's fuzzy inference system has considered with two inputs, one output and totally 49 rules instead of the dynamic equivalent part. Figure 3 is shown error-based fuzzy sliding mode controller. In this method a model free Mamdani's fuzzy inference system has considered based on error-based fuzzy logic controller instead of equivalent control.

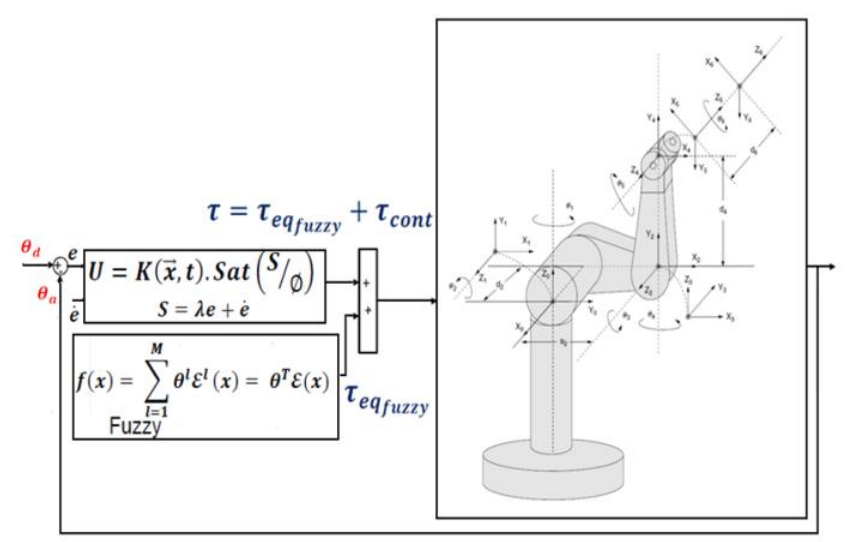

Fig. 3: Fuzzy Sliding Mode Controller

In fuzzy sliding mode controller the PD-sliding surface is defined as follows:

$$
S=\dot{e}+\lambda_{1} e
$$

where $\lambda_{1}=\operatorname{diag}\left[\lambda_{11}, \lambda_{12}, \lambda_{13}\right]$. The time derivative of $\mathrm{S}$ is computed;

$$
\dot{S}=\ddot{q}_{d}+\lambda_{1} \dot{e}
$$

Based on Figure 3, the fuzzy error-based sliding mode controller's output is written;

$$
\hat{\boldsymbol{\tau}}=\tau_{e q_{f u z z y}}+\tau_{s w}
$$


Based on fuzzy logic methodology

$$
f(x)=U_{f u z z y}=\sum_{l=1}^{M} \theta^{T} \zeta(x)
$$

Where $\boldsymbol{\theta}^{\boldsymbol{T}}$ is adjustable parameter (gain updating factor) and $\boldsymbol{\zeta}(\boldsymbol{x})$ is defined by

$$
\zeta(x)=\frac{\sum_{i} \mu\left(x_{i}\right) x_{i}}{\sum_{i} \mu\left(x_{i}\right)}
$$

Where $\boldsymbol{\mu}\left(\boldsymbol{x}_{\boldsymbol{i}}\right)$ is membership function. $\boldsymbol{\tau}_{\boldsymbol{f u z z} \boldsymbol{y}}$ is defined as follows;

$$
\tau_{f u z z y}=\sum_{l=1}^{M} \theta^{T} \zeta(x)=\left[M^{-1}(B+C+G)+\dot{S}\right] M
$$

As mentioned in Figure 3: design of error-based fuzzy instead of equivalent part based on Mamdani's fuzzy inference method has four steps , namely, fuzzification, fuzzy rule base and rule evaluation, aggregation of the rule output (fuzzy inference system) and defuzzification.

Fuzzification: the first step in fuzzification is determine inputs and outputs which, it has two inputs $(e, \dot{e})$ and one output $\left(\tau_{f u z z y}\right)$. The inputs are error (e) which measures the difference between desired and actual output position, and the change of error $(\dot{e})$ which measures the difference between desired and actual velocity and output is fuzzy equivalent torque. The second step is chosen an appropriate membership function for inputs and output which, to simplicity in implementation because it is a linear function with regard to acceptable performance triangular membership function is selected in this research as shown in Figure 4. The third step is chosen the correct labels for each fuzzy set which, in this research namely as linguistic variable. Based on experience knowledge the linguistic variables for error (e) are; Negative Big (NB), Negative Medium (NM), Negative Small (NS), Zero (Z), Positive Small (PS), Positive Medium (PM), Positive Big (PB), and based on literature [40] and experience knowledge it is quantized into thirteen levels represented by: $-1,-0.83,-0.66,-0.5,-0.33,-0.16,0$, $0.16,0.33,0.5,0.66,0.83,1$ the linguistic variables for change of error $(\dot{e})$ are; Fast Left (FL), Medium Left (ML), Slow Left (SL),Zero (Z), Slow Right (SR), Medium Right (MR), Fast Right (FR), and it is quantized in to thirteen levels represented by: $-6,-5$, $0.4,-3,-2,-1,0,1,2,3,4,5,6$, and the linguistic variables to find the output are; Large Left (LL), Medium Left (ML), Small Left (SL), Zero (Z), Small Right (SR), Medium Right (MR), Large Right (LR) and it is quantized in to thirteen levels represented by: -85 , 70.8, -56.7, -42.5, -28.3, -14.2, 0, 14.2, 28.3, 42.5, 56.7, $70.8,85$.

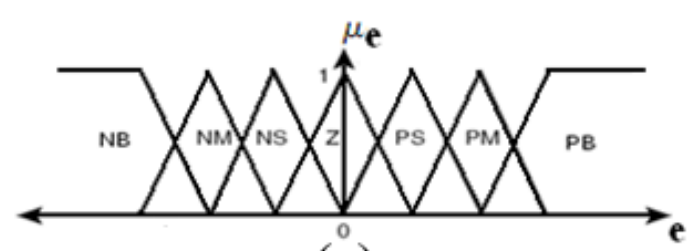

(a)

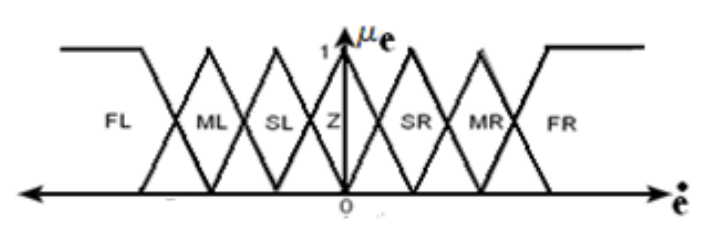

(b)

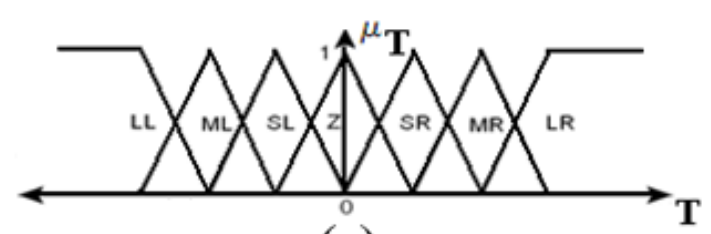

(c)

Fig. 4: Membership function and Linguistic variable

Fuzzy rule base and rule evaluation: the first step in rule base and evaluation is to provide a least structured method to derive the fuzzy rule base which, expert experience and control engineering knowledge is used because this method is the least structure of the other one and the researcher derivation the fuzzy rule base from the knowledge of system operate and/or the classical controller. Design the rule base of fuzzy inference system can play important role to design the best performance of fuzzy sliding mode controller, that to calculate the fuzzy rule base the researcher is used to heuristic method which, it is based on the behavior of the control of robot manipulator. The complete rule base for this controller is shown in Table 1. Rule evaluation focuses on operation in the antecedent of the fuzzy rules in fuzzy sliding mode controller. This part is used $A N D / O R$ fuzzy operation in antecedent part which

\begin{tabular}{|c|c|c|c|c|c|c|c|c|}
\hline \multicolumn{8}{|c|}{ Decrease the overshoot } & \\
\hline$e^{e}$ & FL & ML & SL & $\mathbf{Z}$ & SR & MR & FR & \\
\hline NB & LL & LL & LL & ML & SL & SL & $\mathbf{Z}$ & \\
\hline NM & LL & ML & ML & ML & SL & $\mathbf{Z}$ & SR & \\
\hline NS & LL & ML & SL & SL & $\mathbf{Z}$ & SR & MR & $e$ \\
\hline $\mathbf{Z}$ & LL & ML & SL & $\mathbf{Z}$ & SR & MR & LR & \\
\hline PS & ML & SL & $\mathbf{Z}$ & SR & SR & MR & LR & \\
\hline PM & SL & $\mathbf{Z}$ & SR & MR & MR & MR & LR & \\
\hline PB & $\mathbf{Z}$ & SR & SR & MR & LR & LR & LR & \\
\hline
\end{tabular}
$A N D$ operation is used.

Table 1: Modified Fuzzy Sliding Mode Rule Table

Aggregation of the rule output (Fuzzy inference): Max-Min aggregation is used in this work. 
Defuzzification: The last step to design fuzzy inference in our fuzzy sliding mode controller is defuzzification. This part is used to transform fuzzy set to crisp set, therefore the input for defuzzification is the aggregate output and the output of it is a crisp number. Center of gravity method $(C O G)$ is used in this research.

\section{Gradient Descent Optimization Algoritm and applied to Fuzzy Sliding Mode Controller}

For both sliding mode controller and fuzzy sliding mode controller applications the system performance is sensitive to the sliding surface slope coefficient $(\boldsymbol{\lambda})$. For instance, if large value of $\lambda$ is chosen the response is very fast the system is unstable and conversely, if small value of $\lambda$ is considered the response of system is very slow but system is stable. Therefore to have a good response, compute the best value sliding surface slope coefficient is very important. Gradient descent is based on the observation that if the multivariable function $\boldsymbol{F}(\boldsymbol{x})$ is defined and differentiable in a neighborhood of a point $\boldsymbol{a}$, then $\boldsymbol{F}(\boldsymbol{x})$ decreases fastest if one goes from $\boldsymbol{a}$ in the direction of the negative gradient of $\boldsymbol{F}$ at, $\boldsymbol{a}-\boldsymbol{\nabla} \boldsymbol{F}(\boldsymbol{a})$. It follows that, if

$$
\boldsymbol{b}=a-\gamma \nabla F(a)
$$

For $\gamma \rightarrow 0$ a small enough number, then $F(a)<$ $F(b)$. With this observation in mind, one starts with a guess $x_{0}$ for a local minimum of $F$, and considers the sequence $x_{0}, x_{1}, x_{2}, \ldots$. such that

$$
\boldsymbol{X}_{\boldsymbol{n}+\mathbf{1}}=\boldsymbol{X}_{\boldsymbol{n}}-\gamma_{n} \nabla F\left(X_{n}\right), \quad n \geq 0
$$

We have

$$
F\left(X_{0}\right) \geq F\left(X_{1}\right) \geq F\left(X_{2}\right), \geq \cdots
$$

So hopefully the sequence $\left(X_{n}\right)$ converges to the desired local minimum. Note that the value of the step size $\gamma$ is allowed to change at every iteration. With certain assumptions on the function $F$ (for example, $F$ convex and $\nabla F$ Lipschitz) and particular choices of $\gamma($ e.g., chosen via a line search that satisfies the Wolfe conditions), convergence to a local minimum can be guaranteed. When the function $F$ is convex, all local minima are also global minima, so in this case gradient descent can converge to the global solution.

\section{Design Fuzzy Tuning Based Gradient Descent Optimization Fuzzy Sliding Mode Controller}

In most of industrial robot manipulators, controllers are still usually classical linear, but the manipulator dynamics is highly nonlinear and have uncertain or variation in parameters (e.g., structure and unstructured), as a result design a classical linear controllers for this system is very difficult and sometimes impossible. The first solution is to make the robust algorithm in order to reduce the uncertainty problems in a limit variation (e.g. sliding mode controller and computed torque like controller). Conversely the first solution is used in many applications it has some limitations such as nonlinear dynamic part in controller. The second solution is applied artificial intelligence method (e.g., fuzzy logic) in conventional nonlinear method to reduce or eliminate the challenges. However the second solution is a superior to reduce or eliminate the dynamic nonlinear part with respect to have stability and fairly good robustness but it has a robust in a limit variation. The third solution is used the on-line fuzzy sliding mode controller (e.g., fuzzy-based tuning sliding surface slope in error-based fuzzy sliding mode controller). Adaptive (on-line) control is used in systems whose dynamic parameters are varying and need to be training on line. Error-based fuzzy sliding mode controller has difficulty in handling unstructured model uncertainties and this controller's performance is sensitive to sliding surface slope coefficient. It is possible to solve above challenge by combining fuzzy-based tuning method and errorbased fuzzy sliding mode controller which this methodology can help to improve system's tracking performance by on-line tuning (fuzzy-based tuning) method. Based on above discussion, compute the best value of sliding surface slope coefficient has played important role to improve system's tracking performance especially the system parameters are unknown or uncertain. This problem is solved by tuning the surface slope coefficient $(\boldsymbol{\lambda})$ of the error-based fuzzy sliding mode controller continuously in real-time. In this methodology, the system's performance is improved with respect to the classical sliding mode controller and error-based fuzzy sliding mode controller. Figure 5 shows the fuzzy-based tuning gradient descent optimal fuzzy sliding mode controller.

To adjust the sliding surface slope coefficient we define $\hat{f}(x \mid \lambda)$ as the fuzzy based tuning;

$$
\hat{f}(x \mid \lambda)=\lambda^{T} \zeta(x)
$$

If minimum error $\left(\boldsymbol{\lambda}^{*}\right)$ is defined by;

$$
\lambda^{*}=\arg \min [(\sup \mid \hat{f}(x \mid \lambda)-f(x))]
$$

where $\lambda^{T}$ is adjusted by an adaption law and this law is designed to minimize the error's parameters of $\boldsymbol{\lambda}-\boldsymbol{\lambda}^{*}$. adaption law in fuzzy-based tuning gradient descent error-based fuzzy sliding mode controller is used to adjust the sliding surface slope coefficient online in uncertain system. Fuzzy-based tuning part is a supervisory controller based on Mamdani's fuzzy logic methodology. 


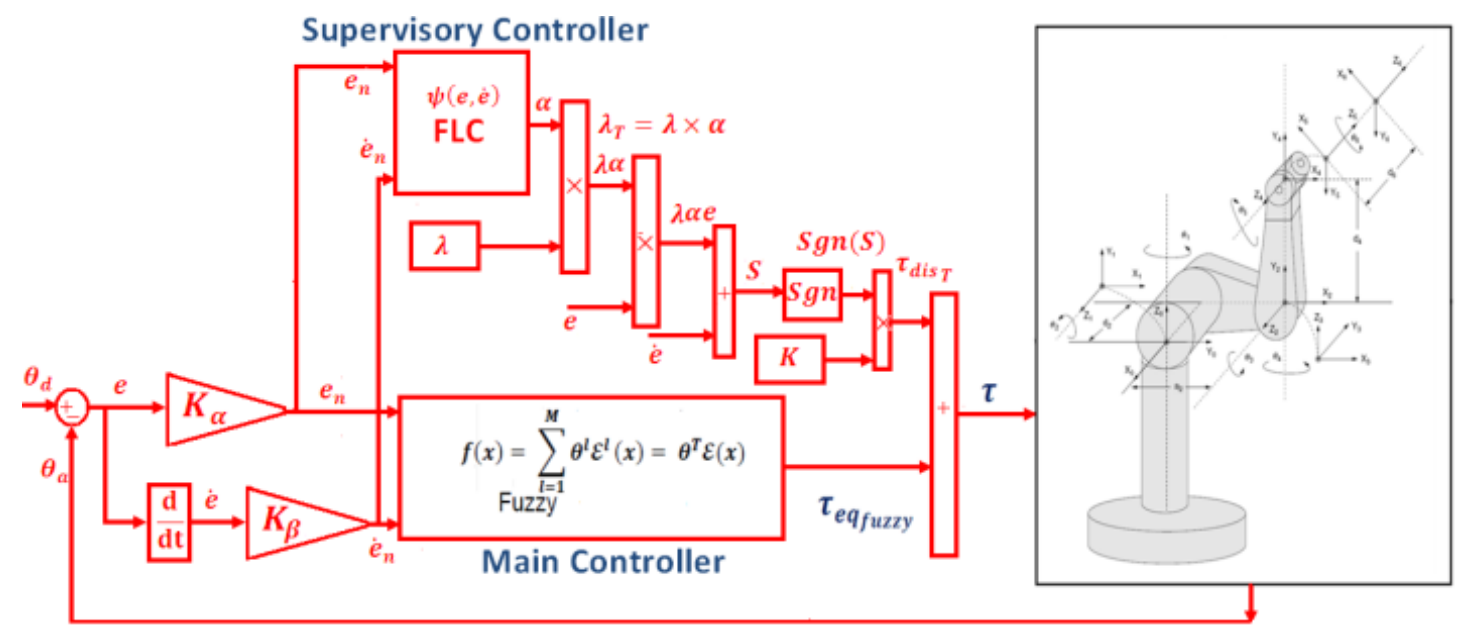

Fig. 5: Fuzzy based tuning gradient descent optimal fuzzy sliding mode controller

\section{Results and Discussion}

Fuzzy-based tuning error-based fuzzy sliding mode controller (FTFSMC) and fuzzy based tuning gradient descent optimal algorithm fuzzy sliding mode controller (GDA) were tested to Step response trajectory. In this simulation, to control position of PUMA robot manipulator the first, second, and third joints are moved from home to final position without and with external disturbance. The simulation was implemented in MATLAB/SIMULINK environment. These systems are tested by band limited white noise with a predefined
$40 \%$ of relative to the input signal amplitude. This type of noise is used to external disturbance in continuous and hybrid systems and applied to nonlinear dynamic of these controllers.

GDA Fuzzy Sliding Mode Controller Optimization: in GDA fuzzy sliding mode controller; controllers performance are depended on the gain updating factor $(K)$ and sliding surface slope coefficient $(\lambda)$. These two coefficients are computed by GDA optimization; Figure 6.

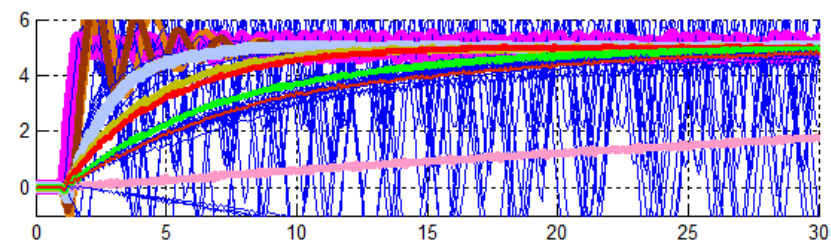

Fig. 6: Gradient descent optimization in fuzzy sliding mode controller

Tracking performances: In error-based fuzzy sliding mode controller; the performance is depended on the gain updating factor $(K)$ and sliding surface slope coefficient $(\lambda)$. These two coefficients are computed by gradient descent optimization. Figure 7 shows tracking performance in FTFSMC and GDA without disturbance for step trajectory.
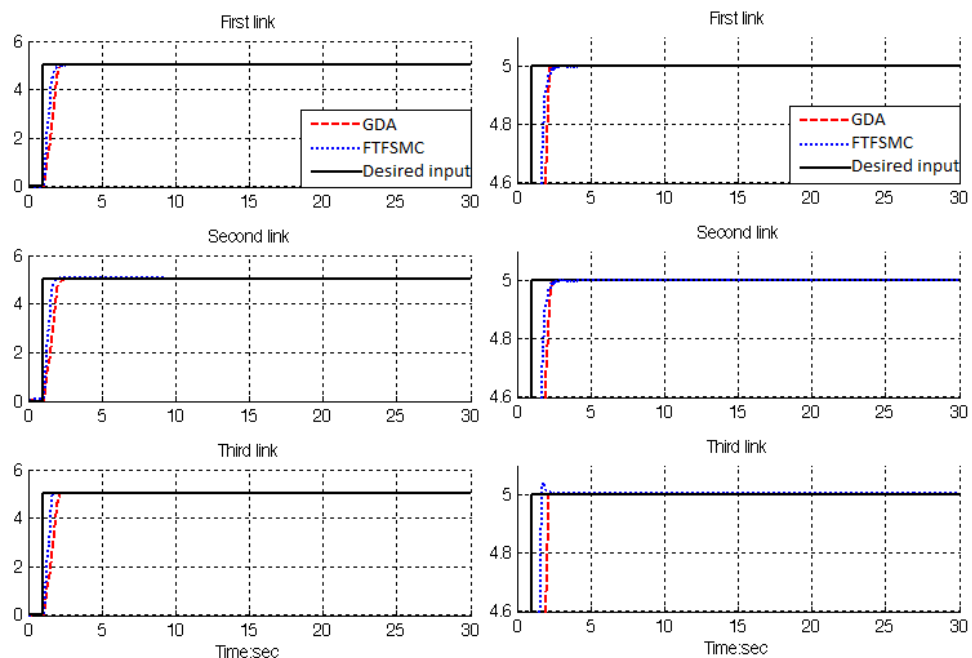

Fig. 7: Fuzzy error based tuning gradient descent optimal FSMC vs. Fuzzy error based tuning FSMC 
Based on Figure 7 it is observed that, the overshoot in FTFSMC's is $1 \%$ and in GDA's is $0 \%$.

Disturbance rejection: Figure 8 is shown the power disturbance elimination in GDA and FTFSMC with disturbance for step trajectory. The disturbance rejection is used to test the robustness comparisons of these controllers for step trajectory. A band limited white noise with predefined of $40 \%$ the power of input signal value is applied to the step trajectory. It found fairly fluctuations in trajectory responses. Based on Figure 8; by comparing step response trajectory with $40 \%$ disturbance of relative to the input signal amplitude in FTFSMC and GDA, GDA's overshoot about (0.5\%) is lower than FTFSMC's (1.2\%). Besides the Steady State and RMS error in FTFSMC and GDA it is observed that, error performances in FTFSMC (Steady State error =1.08e-6 and RMS error=1.5e-6) are about higher than GDA's (Steady State error=0.8e-6 and RMS error=1.1e-6).
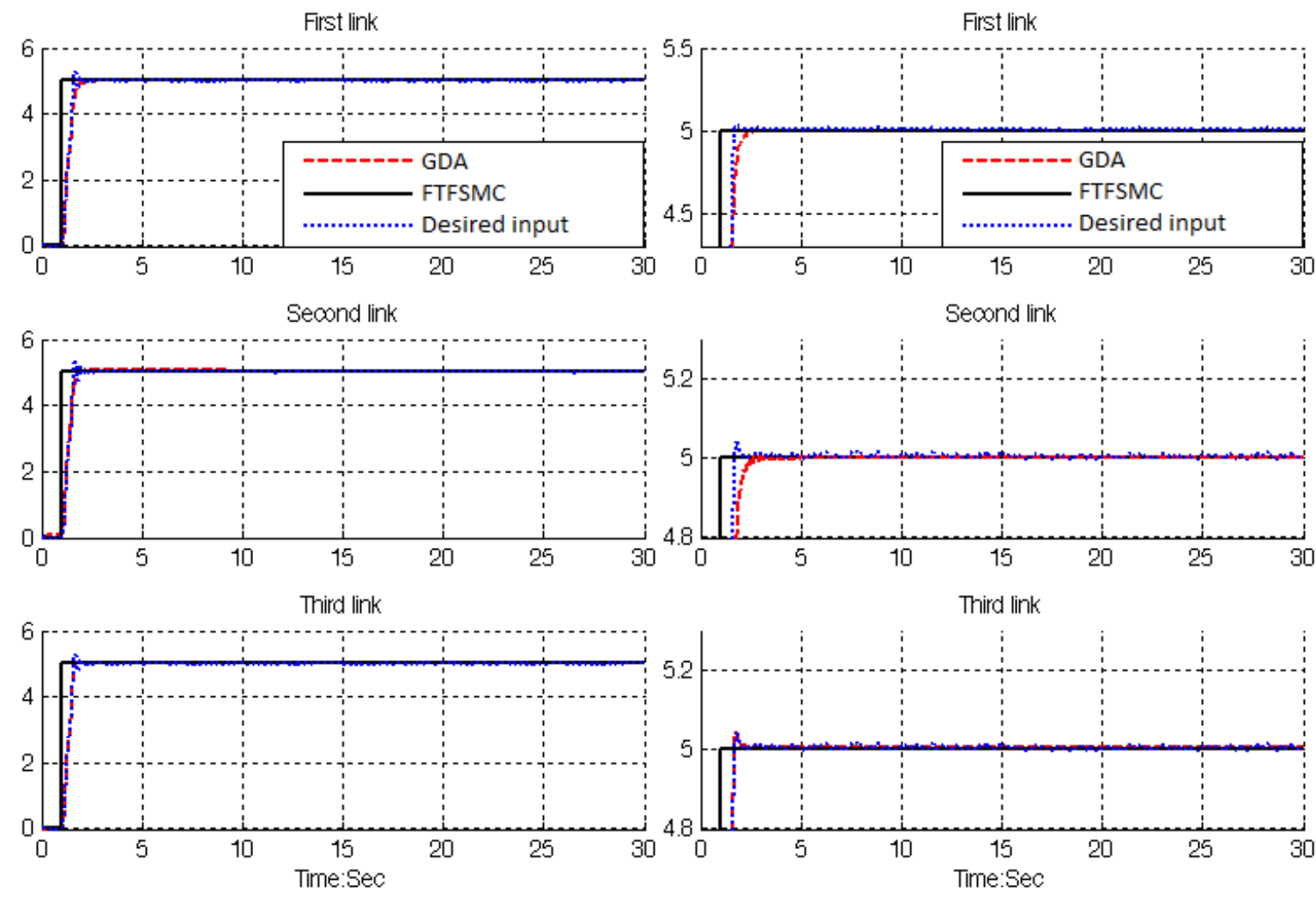

Fig. 8: Fuzzy error based tuning gradient descent optimal FSMC vs. Fuzzy error based tuning FSMC: in presence of $40 \%$ disturbance

Torque performance: Figures 9 and 10 have indicated the power of chattering rejection in GDA and FTFSMC with $40 \%$ disturbance and without disturbance.

Figure 9 shows torque performance for first three links PUMA robot manipulator in GDA and FTFSMC without disturbance. Based on Figure 9, GDA and FTFSMC give considerable torque performance in certain system and both of controllers eliminate the chattering phenomenon in this situation. Figure 10 have indicated the robustness in torque performance for first three links PUMA robot manipulator in GDA and FTFSMC in presence of $40 \%$ disturbance. Based on Figure 10, it is observed that both of two controllers have oscillation. This is mainly because pure SMC with saturation function and fuzzy sliding mode controller with saturation function are robust but they have limitation in presence of external disturbance.
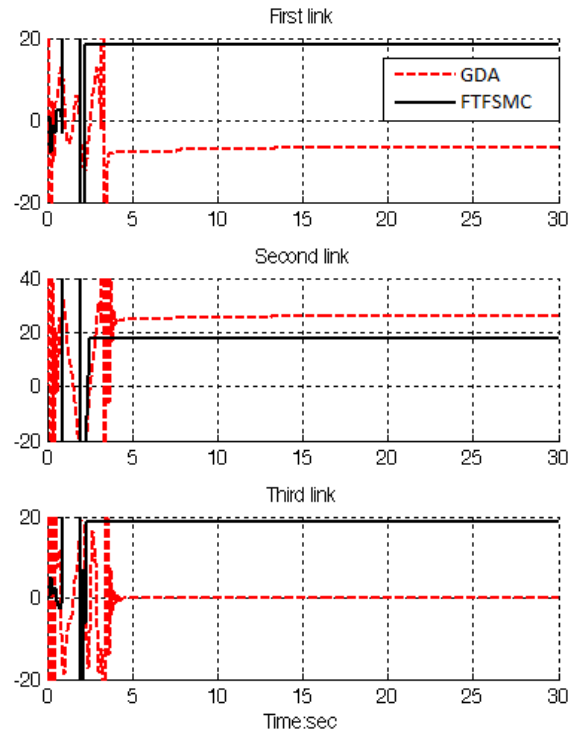

Fig. 9: Fuzzy error based tuning gradient descent optimal FSMC vs. Fuzzy error based tuning FSMC: Torque performance 

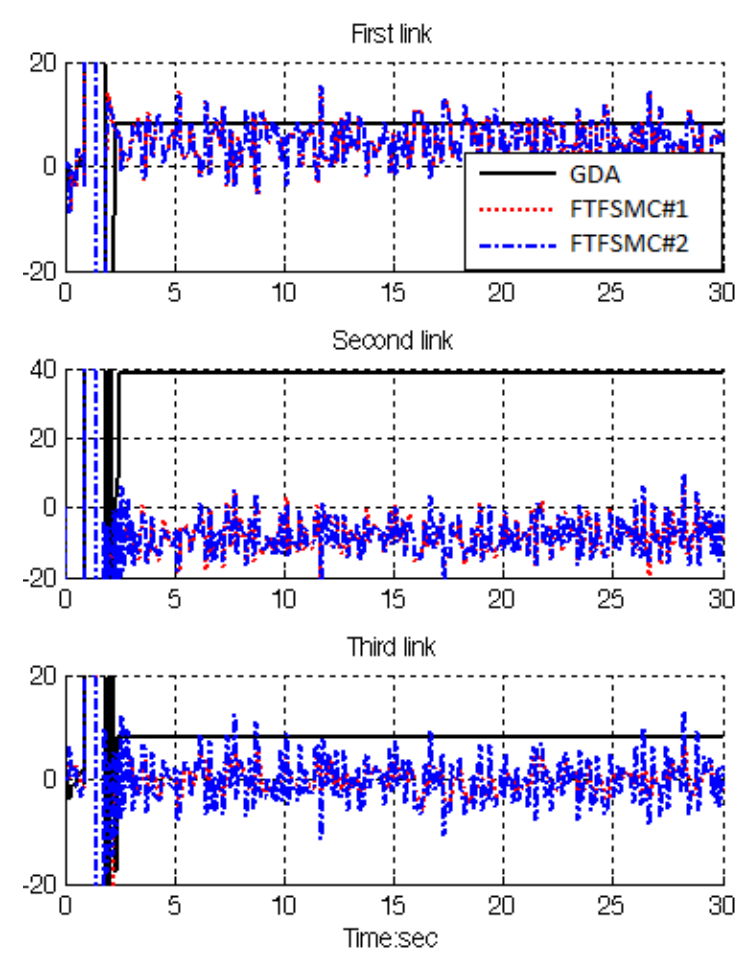

Fig. 10: Fuzzy error based tuning gradient descent optimal FSMC vs. Fuzzy error based tuning FSMC: Torque performance with noise

Based on Figure 10 it is observed that, however fuzzy tuning error-based fuzzy sliding mode controller (FTFSMC) is a model-free controller that eliminate the nonlinear dynamic equivalent formulation by fuzzy rule base but it has significant torque performance (chattering phenomenon) in presence of uncertainty and external disturbance if the sliding surface slope optimized. The GDA gives significant steady state error performance when compared to FTFSMC. When applied $40 \%$ disturbances in GDA the RMS error increased approximately $0.0164 \%$ (percent of increase the GDA RMS error= $\frac{(40 \% \text { disturbance } R M S \text { error }}{\text { no disturbanceRMS error }}=$ $\frac{1.8 e-12}{1.1 e-12}=0.0164 \%$ ) and in FTFSMC the RMS error increased approximately $6.9 \%$ (percent of increase the FSMC RMS error $=\frac{(40 \% \text { disturbance } \text { RMS error }}{\text { no disturbance } R M S \text { error }}=\frac{0.69 e-4}{1 e-7}=$ $6.9 \%)$.

\section{Conclusion}

Refer to this research, a position fuzzy-based tuning gradient descent optimization error-based fuzzy sliding mode controller (GDA) is proposed for PUMA robot manipulator. The first problem of the pure sliding mode controller with switching function was chattering phenomenon in certain and uncertain systems. The nonlinear equivalent dynamic problem in uncertain system is solved by using fuzzy logic theory. To eliminate the PUMA robot manipulator system's dynamic, 49 rules Mamdani inference system is design and applied to sliding mode methodology. This methodology is based on applied fuzzy logic in equivalent nonlinear dynamic part to estimate unknown parameters. The results demonstrate that the error-based fuzzy sliding mode controller is a model-free controllers which works well in certain and partly uncertain system. Pure sliding mode controller and error-based fuzzy sliding mode controller have difficulty in handling unstructured model uncertainties. It is possible to solve this problem by combining error-based fuzzy sliding mode controller and fuzzy-based tuning. Since the sliding surface gain $(\lambda)$ is adjusted by gradient descent optimization method, it is nonlinear and continuous. In tuning online the sliding surface slope updating factor $(\alpha)$ of fuzzy-based tuning part can be changed with the changes in error and change of error rate between half to one. Sliding surface gain is adapted on-line by sliding surface slope updating factor. In pure sliding mode controller and error-based fuzzy sliding mode controller the sliding surface gain is chosen by trial and error, which means pure sliding mode controller and errorbased fuzzy sliding mode controller have to have a prior knowledge of the system uncertainty. If the knowledge is not available error performance and chattering phenomenon are go up. In fuzzy-based tuning errorbased fuzzy sliding mode controller the sliding surface gain are optimized by gradient descent. The stability and convergence of the fuzzy-based tuning error-based fuzzy sliding mode controller based on switching function is guarantee and proved by the Lyapunov method. The simulation results exhibit that the fuzzybased tuning gradient descent optimal error-based fuzzy sliding mode controller works well in various situations.

\section{Acknowledgment}

The authors would like to thank the anonymous reviewers for their careful reading of this paper and for their helpful comments. This work was supported by the SSP Research and Development Corporation Program of Iran under grant no. 2011-Persian Gulf-1A.

\section{References}

[1] T. R. Kurfess, Robotics and automation handbook: CRC, 2005.

[2] J. J. E. Slotine and W. Li, Applied nonlinear control vol. 461: Prentice hall Englewood Cliffs, NJ, 1991.

[3] K. Ogata, Modern control engineering: Prentice Hall, 2009.

[4] B. Siciliano and O. Khatib, Springer handbook of robotics: Springer-Verlag New York Inc, 2008.

[5] I. Boiko, L. Fridman, A. Pisano and E. Usai, "Analysis of chattering in systems with secondorder sliding modes," IEEE Transactions on Automatic Control, No. 11, vol. 52,pp. 2085-2102, 2007. 
[6] J. Wang, A. Rad and P. Chan, "Indirect adaptive fuzzy sliding mode control: Part I: fuzzy switching," Fuzzy Sets and Systems, No. 1, vol. 122,pp. 21-30, 2001.

[7] V. Utkin, "Variable structure systems with sliding modes," Automatic Control, IEEE Transactions on, No. 2, vol. 22, pp. 212-222, 2002.

[8] J. J. Slotine and S. Sastry, "Tracking control of non-linear systems using sliding surfaces, with application to robot manipulators $\dagger, "$ International Journal of Control, No. 2, vol. 38, pp. 465-492, 1983.

[9] P. Kachroo and M. Tomizuka, "Chattering reduction and error convergence in the slidingmode control of a class of nonlinear systems," Automatic Control, IEEE Transactions on, No. 7, vol. 41, pp. 1063-1068, 2002.

[10] H. Elmali and N. Olgac, "Implementation of sliding mode control with perturbation estimation (SMCPE)," Control Systems Technology, IEEE Transactions on, No. 1, vol. 4, pp. 79-85, 2002.

[11] Farzin Piltan , N. Sulaiman, Zahra Tajpaykar, Payman Ferdosali, Mehdi Rashidi, "Design Artificial Nonlinear Robust Controller Based on CTLC and FSMC with Tunable Gain," International Journal of Robotic and Automation, 2 (3): 205-220, 2011.

[12] Farzin Piltan, A. R. Salehi and Nasri B Sulaiman.," Design artificial robust control of second order system based on adaptive fuzzy gain scheduling," world applied science journal (WASJ), 13 (5): 1085-1092, 2011.

[13] Farzin Piltan, N. Sulaiman, Atefeh Gavahian, Samira Soltani, Samaneh Roosta, "Design Mathematical Tunable Gain PID-Like Sliding Mode Fuzzy Controller with Minimum Rule Base," International Journal of Robotic and Automation, 2 (3): 146-156, 2011.

[14] Farzin Piltan , A. Zare, Nasri B. Sulaiman, M. H. Marhaban and R. Ramli, , “A Model Free Robust Sliding Surface Slope Adjustment in Sliding Mode Control for Robot Manipulator," World Applied Science Journal, 12 (12): 2330-2336, 2011.

[15] Farzin Piltan , A. H. Aryanfar, Nasri B. Sulaiman, M. H. Marhaban and R. Ramli "Design Adaptive Fuzzy Robust Controllers for Robot Manipulator," World Applied Science Journal, 12 (12): $2317-$ 2329, 2011.

[16] Farzin Piltan, N. Sulaiman , Arash Zargari, Mohammad Keshavarz, Ali Badri, "Design PIDLike Fuzzy Controller With Minimum Rule Base and Mathematical Proposed On-line Tunable Gain: Applied to Robot Manipulator," International Journal of Artificial intelligence and expert system, 2 (4):184-195, 2011.
[17] Farzin Piltan, Nasri Sulaiman, M. H. Marhaban and R. Ramli, "Design On-Line Tunable Gain Artificial Nonlinear Controller," Journal of Advances In Computer Research, 2 (4): 75-83, 2011.

[18] Farzin Piltan, N. Sulaiman, Payman Ferdosali, Iraj Assadi Talooki, “ Design Model Free Fuzzy Sliding Mode Control: Applied to Internal Combustion Engine," International Journal of Engineering, 5 (4):302-312, 2011.

[19] Farzin Piltan, N. Sulaiman, Samaneh Roosta, M.H. Marhaban, R. Ramli, "Design a New Sliding Mode Adaptive Hybrid Fuzzy Controller," Journal of Advanced Science \& Engineering Research , 1 (1): 115-123, 2011.

[20] Farzin Piltan, Atefe Gavahian, N. Sulaiman, M.H. Marhaban, R. Ramli, "Novel Sliding Mode Controller for robot manipulator using FPGA," Journal of Advanced Science \& Engineering Research, 1 (1): 1-22, 2011.

[21] Farzin Piltan, N. Sulaiman, A. Jalali \& F. Danesh Narouei, "Design of Model Free Adaptive Fuzzy Computed Torque Controller: Applied to Nonlinear Second Order System," International Journal of Robotics and Automation, 2 (4):232-244, 2011.

[22] Farzin Piltan, N. Sulaiman, Iraj Asadi Talooki, Payman Ferdosali, "Control of IC Engine: Design a Novel MIMO Fuzzy Backstepping Adaptive Based Fuzzy Estimator Variable Structure Control ," International Journal of Robotics and Automation, 2 (5):360-380, 2011.

[23] Farzin Piltan, N. Sulaiman, Payman Ferdosali, Mehdi Rashidi, Zahra Tajpeikar, "Adaptive MIMO Fuzzy Compensate Fuzzy Sliding Mode Algorithm: Applied to Second Order Nonlinear System," International Journal of Engineering, 5 (5): 380398, 2011.

[24] Farzin Piltan, N. Sulaiman, Hajar Nasiri, Sadeq Allahdadi, Mohammad A. Bairami, "Novel Robot Manipulator Adaptive Artificial Control: Design a Novel SISO Adaptive Fuzzy Sliding Algorithm Inverse Dynamic Like Method," International Journal of Engineering, 5 (5): 399-418, 2011.

[25] Farzin Piltan, N. Sulaiman, Sadeq Allahdadi, Mohammadali Dialame, Abbas Zare, "Position Control of Robot Manipulator: Design a Novel SISO Adaptive Sliding Mode Fuzzy PD Fuzzy Sliding Mode Control," International Journal of Artificial intelligence and Expert System, 2 (5):208-228, 2011.

[26] Farzin Piltan, SH. Tayebi HAGHIGHI, N. Sulaiman, Iman Nazari, Sobhan Siamak, "Artificial Control of PUMA Robot Manipulator: A-Review of Fuzzy Inference Engine And Application to Classical Controller ," International 
Journal of Robotics and Automation, 2 (5):401-425, 2011.

[27] Farzin Piltan, N. Sulaiman, Abbas Zare, Sadeq Allahdadi, Mohammadali Dialame, "Design Adaptive Fuzzy Inference Sliding Mode Algorithm: Applied to Robot Arm," International Journal of Robotics and Automation , 2 (5): 283-297, 2011.

[28] Farzin Piltan, Amin Jalali, N. Sulaiman, Atefeh Gavahian, Sobhan Siamak, "Novel Artificial Control of Nonlinear Uncertain System: Design a Novel Modified PSO SISO Lyapunov Based Fuzzy Sliding Mode Algorithm ," International Journal of Robotics and Automation, 2 (5): 298316, 2011.

[29] Farzin Piltan, N. Sulaiman, Amin Jalali, Koorosh Aslansefat, "Evolutionary Design of Mathematical tunable FPGA Based MIMO Fuzzy Estimator Sliding Mode Based Lyapunov Algorithm: Applied to Robot Manipulator," International Journal of Robotics and Automation, 2 (5):317-343, 2011.

[30] Farzin Piltan, N. Sulaiman, Samaneh Roosta, Atefeh Gavahian, Samira Soltani, "Evolutionary Design of Backstepping Artificial Sliding Mode Based Position Algorithm: Applied to Robot Manipulator," International Journal of Engineering, 5 (5):419-434, 2011.

[31] Farzin Piltan, N. Sulaiman, S.Soltani, M. H. Marhaban \& R. Ramli, "An Adaptive sliding surface slope adjustment in PD Sliding Mode Fuzzy Control for Robot Manipulator," International Journal of Control and Automation , 4 (3): 65-76, 2011.

[32] Farzin Piltan, N. Sulaiman, Mehdi Rashidi, Zahra Tajpaikar, Payman Ferdosali, "Design and Implementation of Sliding Mode Algorithm: Applied to Robot Manipulator-A Review ," International Journal of Robotics and Automation, 2 (5):265-282, 2011.

[33] Farzin Piltan, N. Sulaiman, Amin Jalali, Sobhan Siamak, and Iman Nazari, "Control of Robot Manipulator: Design a Novel Tuning MIMO Fuzzy Backstepping Adaptive Based Fuzzy Estimator Variable Structure Control ," International Journal of Control and Automation, 4 (4):91-110, 2011.

[34] Farzin Piltan, N. Sulaiman, Atefeh Gavahian, Samaneh Roosta, Samira Soltani, "On line Tuning Premise and Consequence FIS: Design Fuzzy Adaptive Fuzzy Sliding Mode Controller Based on Lyaponuv Theory," International Journal of Robotics and Automation, 2 (5):381-400, 2011.

[35] Farzin Piltan, N. Sulaiman, Samaneh Roosta, Atefeh Gavahian, Samira Soltani, "Artificial Chattering Free on-line Fuzzy Sliding Mode Algorithm for Uncertain System: Applied in Robot
Manipulator," International Journal of Engineering, 5 (5):360-379, 2011.

[36] Farzin Piltan, N. Sulaiman and I.AsadiTalooki, "Evolutionary Design on-line Sliding Fuzzy Gain Scheduling Sliding Mode Algorithm: Applied to Internal Combustion Engine," International Journal of Engineering Science and Technology, 3 (10):7301-7308, 2011.

[37] Farzin Piltan, Nasri B Sulaiman, Iraj Asadi Talooki and Payman Ferdosali.," Designing On-Line Tunable Gain Fuzzy Sliding Mode Controller Using Sliding Mode Fuzzy Algorithm: Applied to Internal Combustion Engine," world applied science journal (WASJ), 15 (3): 422-428, 2011.

[38] B. K. Yoo and W. C. Ham, "Adaptive control of robot manipulator using fuzzy compensator," Fuzzy Systems, IEEE Transactions on, No. 2, vol. 8, pp. 186-199, 2002.

[39] Xiaosong. Lu, "An investigation of adaptive fuzzy sliding mode control for robot manipulator," Carleton university Ottawa,2007.

[40] S. Lentijo, S. Pytel, A. Monti, J. Hudgins, E. Santi and G. Simin, "FPGA based sliding mode control for high frequency power converters," IEEE Conference, 2004, pp. 3588-3592.

[41] B. S. R. Armstrong, "Dynamics for robot control: friction modeling and ensuring excitation during parameter identification," 1988.

[42] C. L. Clover, "Control system design for robots used in simulating dynamic force and moment interaction in virtual reality applications," 1996.

[43] K. R. Horspool, Cartesian-space Adaptive Control for Dual-arm Force Control Using Industrial Robots: University of New Mexico, 2003.

[44] B. Armstrong, O. Khatib and J. Burdick, "The explicit dynamic model and inertial parameters of the PUMA 560 arm," IEEE Conference, 2002, pp. 510-518.

[45] P. I. Corke and B. Armstrong-Helouvry, "A search for consensus among model parameters reported for the PUMA 560 robot," IEEE Conference, 2002, pp. 1608-1613.

[46] Farzin Piltan, N. Sulaiman, M. H. Marhaban, Adel Nowzary, Mostafa Tohidian," "Design of FPGA based sliding mode controller for robot manipulator," International Journal of Robotic and Automation, 2 (3): 183-204, 2011.

[47] I. Eksin, M. Guzelkaya and S. Tokat, "Sliding surface slope adjustment in fuzzy sliding mode controller," Mediterranean Conference, 2002, pp. 160-168.

[48] Samira Soltani \& Farzin Piltan, "Design Artificial Nonlinear Controller Based on Computed Torque 
like Controller with Tunable Gain". World Applied Science Journal,14 (9): 1306-1312, 2011.

[49] Farzin Piltan, H. Rezaie, B. Boroomand, Arman Jahed," Design robust back stepping online tuning feedback linearization control applied to IC engine," International Journal of Advance Science and Technology, 42: 183-204, 2012.

[50] Farzin Piltan, I. Nazari, S. Siamak, P. Ferdosali ,"Methodology of FPGA-based mathematical error-based tuning sliding mode controller" International Journal of Control and Automation, 5(1): 89-110, 2012.

[51] Farzin Piltan, M. A. Dialame, A. Zare, A. Badri ,'Design Novel Lookup table changed Auto Tuning FSMC: Applied to Robot Manipulator" International Journal of Engineering, 6(1): 25-40, 2012.

[52] Farzin Piltan, B. Boroomand, A. Jahed, H. Rezaie ,"Methodology of Mathematical ErrorBased Tuning Sliding Mode Controller" International Journal of Engineering, 6(2): 96-112, 2012.

[53] Farzin Piltan, F. Aghayari, M. R. Rashidian, M. Shamsodini, "A New Estimate Sliding Mode Fuzzy Controller for Robotic Manipulator" International Journal of Robotics and Automation, 3(1): 45-58, 2012.

[54] Farzin Piltan, M. Keshavarz, A. Badri, A. Zargari , "Design novel nonlinear controller applied to robot manipulator: design new feedback linearization fuzzy controller with minimum rule base tuning method" International Journal of Robotics and Automation, 3(1): 1-18, 2012.

[55] Piltan, F., et al. "Design sliding mode controller for robot manipulator with artificial tunable gain". Canaidian Journal of pure and applied science, 5 (2), 1573-1579, 2011.

[56] Farzin Piltan, A. Hosainpour, E. Mazlomian, M.Shamsodini, M.H Yarmahmoudi. "Online Tuning Chattering Free Sliding Mode Fuzzy Control Design: Lyapunov Approach" International Journal of Robotics and Automation, 3(3): 2012.

[57] Farzin Piltan , M.H. Yarmahmoudi, M. Shamsodini, E.Mazlomian, A.Hosainpour. " PUMA-560 Robot Manipulator Position Computed Torque Control Methods Using MATLAB/SIMULINK and Their Integration into Graduate Nonlinear Control and MATLAB Courses" International Journal of Robotics and Automation, 3(3): 2012.

[58] Farzin Piltan, R. Bayat, F. Aghayari, B. Boroomand. "Design Error-Based Linear ModelFree Evaluation Performance Computed Torque Controller" International Journal of Robotics and Automation, 3(3): 2012.
[59] Farzin Piltan, S. Emamzadeh, Z. Hivand, F. Shahriyari \& Mina Mirazaei ." PUMA-560 Robot Manipulator Position Sliding Mode Control Methods Using MATLAB/SIMULINK and Their Integration into Graduate/Undergraduate Nonlinear Control, Robotics and MATLAB Courses" International Journal of Robotics and Automation, 3(3): 2012.

[60] Farzin Piltan, J. Meigolinedjad, S. Mehrara, S. Rahmdel. " Evaluation Performance of $2^{\text {nd }}$ Order Nonlinear System: Baseline Control Tunable Gain Sliding Mode Methodology" International Journal of Robotics and Automation, 3(3): 2012.

[61] Farzin Piltan, M. Mirzaie, F. Shahriyari, Iman Nazari \& S. Emamzadeh." Design Baseline Computed Torque Controller" International Journal of Engineering, 3(3): 2012.

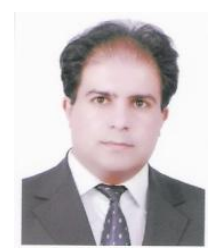

Farzin Piltan was born on 1975, Shiraz, Iran. In 2004 he is jointed the research and development company, SSP Co, Shiraz, Iran. In addition to 7 textbooks, Farzin Piltan is the main author of more than 50 scientific papers in refereed journals. He is editorial board of international journal of control and automation (IJCA), editorial board of IAES international journal of robotics and automation, editorial board of International Journal of Reconfigurable and Embedded Systems and reviewer of (CSC) international journal of robotics and automation. His main areas of research interests are nonlinear control, artificial control system and applied to FPGA, robotics and artificial nonlinear control and IC engine modelling and control.

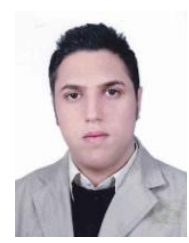

Bamdad Boroomand is an expert Mechanical engineer of SSP Co. research center. His current research interests are including modeling, control \& automation and robotics.

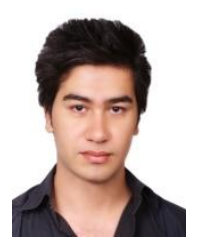

Arman Jahed is an expert Mechanical engineer of SSP Co. research center. His current research interests are including modeling, control \& automation and robotics.

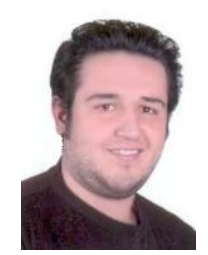

Hossein Rezaie is an expert Mechanical engineer of SSP Co. research center. His current research interests are including modeling, control \& automation and robotics. 